PROCEEDINGS OF THE

AMERICAN MATHEMATICAL SOCIETY

Volume 132, Number 7, Pages 2067-2073

S 0002-9939(04)07327-7

Article electronically published on January 29, 2004

\title{
UNIQUENESS OF DILATION INVARIANT NORMS
}

\author{
E. MORENO AND A. R. VILLENA
}

(Communicated by N. Tomczak-Jaegermann)

\begin{abstract}
Let $\delta_{a}$ be a nontrivial dilation. We show that every complete norm $\|\cdot\|$ on $L^{1}\left(\mathbb{R}^{N}\right)$ that makes $\delta_{a}$ from $\left(L^{1}\left(\mathbb{R}^{N}\right),\|\cdot\|\right)$ into itself continuous is equivalent to $\|\cdot\|_{1} . \delta_{a}$ also determines the norm of both $C_{0}\left(\mathbb{R}^{N}\right)$ and $L^{p}\left(\mathbb{R}^{N}\right)$ with $1<p<\infty$ in a weaker sense. Furthermore, we show that even all the dilations do not determine the norm on $L^{\infty}\left(\mathbb{R}^{N}\right)$.
\end{abstract}

\section{INTRODUCTION}

The uniqueness-of-norm problem is a classical topic in automatic continuity theory, which has been mainly developed in the context of Banach algebras. The most important result in this area is the famous theorem by B. E. Johnson [6] that every semisimple Banach algebra, such as $L^{1}(G)$ for any locally compact group $G$, carries a unique Banach algebra norm. Recently, quite a lot of attention has been paid to the question of whether $L^{p}(G)$ with $1 \leq p \leq+\infty$ carries a unique translation invariant norm. Investigation about this subject started with the seminal paper by K. Jarosz $[5]$ on $L^{1}(\mathbb{R})$ and $L^{p}(\mathbb{T})(1 \leq p \leq \infty)$, and it has been successfully carried out for arbitrary locally compact abelian groups [2], 8] and for some classes of nonabelian groups such as compact groups [3] and Moore groups [4]. It turned out that the norm $\|\cdot\|_{p}$ on $L^{p}(G)$ is intimately connected with the action of translations on $G$, and that the uniqueness of translation invariant norms on $L^{p}(G)$ is closely related to the classical problem of determining whether or not there is a discontinuous translation invariant functional on $L^{p}(G)$.

An essential feature of harmonic analysis is the fact that the Fourier transform has a very simple transformation law under translations. This fact was the key to obtaining the results in the above-mentioned papers. Harmonic analysis in Euclidean spaces, however, is richer because of its connection with several classes of transformations: the dilations and the rotations as well as the translations. In this paper we wish to make quite explicit the further connection between the norm $\|\cdot\|_{p}$ on $L^{p}\left(\mathbb{R}^{N}\right)$ and the action of dilations by proving that every complete norm $\|\cdot\|$ on $L^{1}\left(\mathbb{R}^{N}\right)$ that makes a single nontrivial dilation from $\left(L^{1}\left(\mathbb{R}^{N}\right),\|\cdot\|\right)$ into itself continuous is equivalent to the norm $\|\cdot\|_{1}$. When concerned with the space $L^{p}\left(\mathbb{R}^{N}\right)$ with $1 \leq p<\infty$ we show that every complete norm $\|\cdot\|$ with the property that there exists $a>0$ with $a \neq 1$ such that $\left\|\delta_{a} f\right\|=a^{-\frac{N}{p}}\|f\|$ for each $f \in L^{p}\left(\mathbb{R}^{N}\right)$ is equivalent to $\|\cdot\|_{p}$. Furthermore, we prove that a similar result holds true for $C_{0}\left(\mathbb{R}^{N}\right)$

Received by the editors November 19, 2002 and, in revised form, April 1, 2003.

2000 Mathematics Subject Classification. Primary 46E30, 46H40.

The second author was supported by MCYT Grant BFM2003-01681. 
and that there exists a complete norm $\|\cdot\|$ on $L^{\infty}\left(\mathbb{R}^{N}\right)$ such that $\left\|\delta_{a} f\right\|=\|f\|$ for all $f \in L^{\infty}\left(\mathbb{R}^{N}\right)$ and $a>0$ and that it is not equivalent to the norm $\|\cdot\|_{\infty}$.

Throughout this paper, for any $a>0$ and any function $f: \mathbb{R}^{N} \rightarrow \mathbb{C}$, we write $\delta_{a} f$ to denote the dilation of $f$ defined by $\left(\delta_{a} f\right)(x)=f(a x)$ for each $x \in \mathbb{R}^{N}$.

\section{Preliminaries}

Recall that the separating space $\mathfrak{S}(\Phi)$ of a linear mapping $\Phi$ between Banach spaces $X$ and $Y$ is defined by

$$
\mathfrak{S}(\Phi)=\left\{y \in Y: \exists\left(x_{n}\right) \text { in } X \text { with } \lim x_{n}=0 \text { and } \lim \Phi\left(x_{n}\right)=y\right\} .
$$

By the closed graph theorem, $\mathfrak{S}(\Phi)=\{0\}$ if and only if $\Phi$ is continuous. A standard fact we shall use is that $\Psi \circ \Phi$ is continuous if and only if $\Psi(\mathfrak{S}(\Phi))=0$ whenever $\Psi$ is a continuous linear operator from $Y$ into another Banach space $Z$ ([1] Proposition 5.2.2(ii)]). The fundamental result about the separating space is the stability lemma ([1, Theorem 5.2.5(ii)]), and we require the following variant of that result which can be found in [1, Corolary 5.2.7(i)].

Lemma 1. Let $X$ and $Y$ be Banach spaces, and let $\left(R_{n}\right)$ and $\left(S_{n}\right)$ be sequences of continuous linear operators on $X$ and $Y$, respectively. If $\Phi$ is a linear map from $X$ into $Y$ such that $S_{n} \circ \Phi=\Phi \circ R_{n}$ for each $n \in \mathbb{N}$, then there exists $m \in \mathbb{N}$ such that

$$
\overline{\left(S_{1} \circ \cdots \circ S_{m}\right)(\mathfrak{S}(\Phi))}=\overline{\left(S_{1} \circ \cdots \circ S_{n}\right)(\mathfrak{S}(\Phi))}
$$

for each $n \geq m$.

\section{UNIQUENESS OF NORM ON $L^{1}\left(\mathbb{R}^{N}\right)$}

It is immediate to check that the norm $\|\cdot\|_{1}$ on $L^{1}\left(\mathbb{R}^{N}\right)$ satisfies the following property:

$$
\left\|\delta_{a} f\right\|_{1}=a^{-N}\|f\|_{1}
$$

for all $a>0$ and $f \in L^{1}\left(\mathbb{R}^{N}\right)$. In fact, our purpose is to show that this property characterizes $\|\cdot\|_{1}$ even when restricted to a single nontrivial dilation $\delta_{a}$.

Theorem 1. Let $a>0$ with $a \neq 1$. If $\|\cdot\|$ is a complete norm on $L^{1}\left(\mathbb{R}^{N}\right)$ such that the dilation $\delta_{a}$ from $\left(L^{1}\left(\mathbb{R}^{N}\right),\|\cdot\|\right)$ into itself is continuous, then $\|\cdot\|$ is equivalent to $\|\cdot\|_{1}$.

Proof. We only need to show that the identity map $\Phi$ from $\left(L^{1}\left(\mathbb{R}^{N}\right),\|\cdot\|\right)$ into $\left(L^{1}\left(\mathbb{R}^{N}\right),\|\cdot\|_{1}\right)$ is continuous.

For every $\varepsilon=\left(\varepsilon_{1}, \ldots, \varepsilon_{N}\right) \in\{-1,1\}^{N}$, let $\Psi_{\varepsilon}$ be the linear operator from $\left(L^{1}\left(\mathbb{R}^{N}\right),\|\cdot\|_{1}\right)$ into itself defined by

$$
\left(\Psi_{\varepsilon} f\right)(x)=f\left(\varepsilon_{1} e^{x_{1}}, \ldots, \varepsilon_{N} e^{x_{N}}\right) e^{x_{1}+\cdots+x_{N}}
$$

for all $f \in L^{1}\left(\mathbb{R}^{N}\right)$ and $x=\left(x_{1}, \ldots, x_{N}\right) \in \mathbb{R}^{N}$.

We begin by proving that $\Phi_{\varepsilon}=\Psi_{\varepsilon} \circ \Phi:\left(L^{1}\left(\mathbb{R}^{N}\right),\|\cdot\|\right) \rightarrow\left(L^{1}\left(\mathbb{R}^{N}\right),\|\cdot\|_{1}\right)$ is continuous for each $\varepsilon \in\{-1,1\}^{N}$. Let us denote by $\vartheta$ the continuous function from $\mathbb{R}^{N}$ into $\mathbb{C}$ given by

$$
\vartheta(x)=e^{2 \pi i \ln a\left(x_{1}+\cdots+x_{N}\right)}
$$

for each $x \in \mathbb{R}^{N}$. Let $\left\{t_{n}: n \in \mathbb{N}\right\}$ be a countable dense subset of $\mathbb{R}^{N}$. For every $n \in \mathbb{N}$, set $\lambda_{n}=\vartheta\left(t_{n}\right)$. Let us denote by $R_{n}$ the continuous linear operator from $\left(L^{1}\left(\mathbb{R}^{N}\right),\|\cdot\|\right)$ into itself given by

$$
R_{n} f=a^{N} \delta_{a} f-\lambda_{n} f
$$


for each $f \in L^{1}\left(\mathbb{R}^{N}\right)$, and let us denote by $S_{n}$ the continuous linear operator from $\left(L^{1}\left(\mathbb{R}^{N}\right),\|\cdot\|_{1}\right)$ into itself given by

$$
\left(S_{n} f\right)(x)=f\left(x_{1}+\ln a, \ldots, x_{N}+\ln a\right)-\lambda_{n} f(x)
$$

for all $x \in \mathbb{R}^{N}$ and $f \in L^{1}\left(\mathbb{R}^{N}\right)$. The key facts in the proof are that

$$
\Phi_{\varepsilon} \circ R_{n}=S_{n} \circ \Phi_{\varepsilon} \quad \forall n \in \mathbb{N}
$$

and that

$$
\widehat{S_{n} f}(x)=\left(\vartheta(x)-\lambda_{n}\right) \widehat{f}(x) \quad \forall n \in \mathbb{N}, f \in L^{1}\left(\mathbb{R}^{N}\right), x \in \mathbb{R}^{N},
$$

as is easy to check. Here and subsequently $\widehat{f}$ stands for the Fourier transform of any function $f \in L^{1}\left(\mathbb{R}^{N}\right)$, i.e.,

$$
\widehat{f}(x)=\int_{\mathbb{R}^{N}} e^{-2 \pi i\left(x_{1} t_{1}+\cdots+x_{N} t_{N}\right)} f\left(t_{1}, \ldots, t_{N}\right) d\left(t_{1}, \ldots, t_{N}\right) .
$$

From (1) and Lemma 1 it follows that there exists $m \in \mathbb{N}$ such that

$$
\overline{\left(S_{1} \circ \cdots \circ S_{m}\right)\left(\mathfrak{S}\left(\Phi_{\varepsilon}\right)\right)}=\overline{\left(S_{1} \circ \cdots \circ S_{n}\right)\left(\mathfrak{S}\left(\Phi_{\varepsilon}\right)\right)}
$$

for each $n \geq m$. We now observe that the Fourier transform of every function of the latter set vanishes at $t_{k}$ for $k=1, \ldots, n$. Indeed, on account of (2), we have

$$
\begin{aligned}
{\left[\left(S_{1} \circ \cdots \circ S_{n}\right)(f)\right]^{\wedge}\left(t_{k}\right) } & =\left(\vartheta\left(t_{k}\right)-\lambda_{1}\right) \cdots\left(\vartheta\left(t_{k}\right)-\lambda_{n}\right) \widehat{f}\left(t_{k}\right) \\
& =\left(\lambda_{k}-\lambda_{1}\right) \cdots\left(\lambda_{k}-\lambda_{n}\right) \widehat{f}\left(t_{k}\right)=0
\end{aligned}
$$

for each $f \in L^{1}\left(\mathbb{R}^{N}\right)$. Consequently, for every $f \in \mathfrak{S}\left(\Phi_{\varepsilon}\right)$,

$$
\left(\vartheta\left(t_{k}\right)-\lambda_{1}\right) \cdots\left(\vartheta\left(t_{k}\right)-\lambda_{m}\right) \widehat{f}\left(t_{k}\right)=\left[\left(S_{1} \circ \cdots \circ S_{m}\right)(f)\right]^{\wedge}\left(t_{k}\right)=0 .
$$

Therefore, for every $f \in \mathfrak{S}\left(\Phi_{\varepsilon}\right)$, the function $\left(\vartheta-\lambda_{1}\right) \cdots\left(\vartheta-\lambda_{m}\right) \widehat{f}$ vanishes on the set $\left\{t_{n}: n \in \mathbb{N}\right\}$. From the continuity of the function and the density of the set it may be concluded that

$$
\left(\vartheta-\lambda_{1}\right) \cdots\left(\vartheta-\lambda_{m}\right) \widehat{f}=0 .
$$

Hence $\widehat{f}(x)=0$ whenever $x$ lies in $\mathbb{R}^{N} \backslash \Sigma$, where

$$
\Sigma=\left\{x \in \mathbb{R}^{N}:\left(\vartheta(x)-\lambda_{1}\right) \cdots\left(\vartheta(x)-\lambda_{m}\right)=0\right\} .
$$

Since $\Sigma$ does not have any interior point, we conclude that $\mathbb{R}^{N} \backslash \Sigma$ is dense and hence that $\widehat{f}=0$. We thus get $f=0$, as required.

We are now in a position to show that $\Phi$ is continuous. Since $\Phi_{\varepsilon}$ is continuous, we have

$$
\Psi_{\varepsilon}(\mathfrak{S}(\Phi))=0
$$

for each $\varepsilon \in\{-1,1\}^{N}$. Let $f \in \mathfrak{S}(\Phi)$. Then the function

$$
\left(x_{1}, \ldots, x_{N}\right) \mapsto f\left(\varepsilon_{1} e^{x_{1}}, \ldots, \varepsilon_{N} e^{x_{N}}\right) e^{x_{1}+\cdots+x_{N}}
$$

vanishes almost everywhere; so

$$
\int_{\mathbb{R}^{N}}\left|f\left(\varepsilon_{1} e^{x_{1}}, \ldots, \varepsilon_{N} e^{x_{N}}\right)\right| e^{x_{1}+\cdots+x_{N}} d\left(x_{1}, \ldots, x_{N}\right)=0
$$


for each $\varepsilon \in\{-1,1\}^{N}$. On the other hand, we have

$$
\begin{aligned}
\int_{\mathbb{R}^{N}}|f(t)| d t & =\sum_{\varepsilon \in\{-1,1\}^{N}} \int_{] 0,+\infty\left[{ }^{N}\right.}\left|f\left(\varepsilon_{1} t_{1}, \ldots, \varepsilon_{N} t_{N}\right)\right| d t \\
& =\int_{\mathbb{R}^{N}}\left|f\left(\varepsilon_{1} e^{x_{1}}, \ldots, \varepsilon_{N} e^{x_{N}}\right) e^{x_{1}+\cdots+x_{N}}\right| d\left(x_{1}, \ldots, x_{N}\right)=0,
\end{aligned}
$$

which shows that $f=0$ and hence that $\Phi$ is continuous.

$$
\text { 4. Uniqueness OF NORM ON } C_{0}\left(\mathbb{R}^{N}\right) \text { AND } L^{p}\left(\mathbb{R}^{N}\right)
$$

Note that

$$
\left\|\delta_{a} f\right\|_{\infty}=\|f\|_{\infty}
$$

and that

$$
\left\|\delta_{a} g\right\|_{p}=a^{-\frac{N}{p}}\|g\|_{p}
$$

for all $a>0, f \in C_{0}\left(\mathbb{R}^{N}\right)$, and $g \in L^{p}\left(\mathbb{R}^{N}\right)(1<p<\infty)$. It is to be expected that both spaces $C_{0}\left(\mathbb{R}^{N}\right)$ and $L_{p}\left(\mathbb{R}^{N}\right)$ carry a unique dilation invariant norm in the sense of Theorem 1. Unfortunately, we have not been able to prove such a result. In this section we prove that every complete norm on $C_{0}\left(\mathbb{R}^{N}\right)$ satisfying identity (3i) for some nontrivial dilation $\delta_{a}$ is equivalent to the norm $\|\cdot\|_{\infty}$ and that every complete norm on $L_{p}\left(\mathbb{R}^{N}\right)$ satisfying identity (4) for some nontrivial dilation $\delta_{a}$ is equivalent to $\|\cdot\|_{p}$.

The key point for proving that result is that, for every $a>0$, the group $\mathbb{Z}$ acts on both spaces $C_{0}\left(\mathbb{R}^{N}\right)$ and $L^{p}\left(\mathbb{R}^{N}\right)$ by

$$
k \cdot f=\delta_{a}{ }^{k} f \text { and } k \cdot g=a^{\frac{N k}{p}} \delta_{a}{ }^{k} g
$$

for all $k \in \mathbb{Z}, f \in C_{0}\left(\mathbb{R}^{N}\right)$, and $g \in L^{p}\left(\mathbb{R}^{N}\right)$, respectively. It is immediate that both spaces $\left(C_{0}\left(\mathbb{R}^{N}\right),\|\cdot\|\right)$ and $\left(L^{p}\left(\mathbb{R}^{N}\right),\|\cdot\|\right)$ are Banach $\mathbb{Z}$-modules in the sense of [7, Definition 1.1] with operations given by (5) whenever $\|\cdot\|$ is a complete norm satisfying identity (3) or identity (4), respectively. Let us recall that a Banach $G$ module, for a given locally compact abelian group $G$, is a Banach space $X$ equipped with a mapping $(t, x) \mapsto t \cdot x$ from $G \times X$ into $X$ such that

(i) $x \mapsto t \cdot x$ is linear on $X$ for each $t \in G$;

(ii) $s \cdot(t \cdot x)=(s t) \cdot x$ for all $s, t \in G$ and $x \in X$;

(iii) $e \cdot x=x$ for each $x \in X$, where $e$ stands for the identity of $G$;

(iv) there exists $K>0$ with $\|t \cdot x\| K \leq\|x\|$ for all $t \in G$ and $x \in X$.

A $G$-submodule of $X$ is a closed linear subspace $M$ of $X$ with $G \cdot M \subset M$. The $G$ submodule $M$ is said to be scalar if for each $t \in G$ there is $\lambda(t) \in \mathbb{C}$ with $t \cdot x=\lambda(t) x$ for all $t \in G$ and $x \in M$.

Our proof consists in proving that every scalar $\mathbb{Z}$-submodule of both $\mathbb{Z}$-modules $C_{0}\left(\mathbb{R}^{N}\right)$ and $L^{p}\left(\mathbb{R}^{N}\right)$ (for a given $a \neq 1$ ) is zero and applying the following.

Theorem 2 ([7, Theorem 4.1]). Let $G$ be a locally compact abelian group, let $X$, $Y$ be Banach $G$-modules, and let $\Phi: X \rightarrow Y$ be a $G$-module homomorphism. Then $\mathfrak{S}(\Phi)$ is the direct sum of a finite number of scalar $G$-submodules of $Y$.

Lemma 2. Let $\delta_{a}$ be any nontrivial dilation. Then the following assertions hold:

i. The operator $\delta_{a}$ on $C_{0}\left(\mathbb{R}^{N}\right)$ does not have any eigenvalue. Accordingly, $C_{0}\left(\mathbb{R}^{N}\right)$ does not have any nonzero scalar $\mathbb{Z}$-submodule. 
ii. The operator $\delta_{a}$ on $L^{p}\left(\mathbb{R}^{N}\right)$ with $1 \leq p<\infty$ does not have any eigenvalue. Accordingly, $L^{p}\left(\mathbb{R}^{N}\right)$ does not have any nonzero scalar $\mathbb{Z}$-submodule.

Proof. To obtain a contradiction, suppose that there exist $\lambda \in \mathbb{C}$ and $f \in C_{0}\left(\mathbb{R}^{N}\right)$ with $f \neq 0$ such that $\delta_{a}(f)=\lambda f$.

Since $\delta_{a}$ is an isomorphism from $C_{0}\left(\mathbb{R}^{N}\right)$ onto itself with $\delta_{a}{ }^{-1}=\delta_{a^{-1}}$, we see that $\lambda \neq 0$. On the other hand, we can certainly assume that $a>1$, for if not, we replace $\delta_{a}$ with $\delta_{a^{-1}}$ and $\lambda$ with $\lambda^{-1}$.

Let $x_{0} \in \mathbb{R}^{N} \backslash\{0\}$ such that $f\left(x_{0}\right) \neq 0$. Then

$$
f\left(a^{n} x_{0}\right)=\delta_{a}^{n}(f)\left(x_{0}\right)=\lambda^{n} f\left(x_{0}\right)
$$

for each $n \in \mathbb{N}$. Since $\lim _{n \rightarrow \infty}\left\|a^{n} x_{0}\right\|_{2}=+\infty$ and $\lim _{\|x\|_{2} \rightarrow+\infty} f(x)=0$, it follows that

$$
\lim _{n \rightarrow \infty} \lambda^{n}=\lim _{n \rightarrow \infty} f\left(x_{0}\right)^{-1} f\left(a^{n} x_{0}\right)=0 .
$$

On the other hand, we have

$$
f\left(x_{0}\right)=\lambda^{n} f\left(x_{0} / a^{n}\right)
$$

for each $n \in \mathbb{N}$. Since $f$ is bounded and $\lim _{n \rightarrow \infty} \lambda^{n}=0$, it may be concluded that $f\left(x_{0}\right)=0$, a contradiction.

We now assume that there exist $\lambda \in \mathbb{C}$ and $f \in L^{p}\left(\mathbb{R}^{N}\right)$ such that $\delta_{a} f=\lambda f$. Define $g=|f|^{p}$. Then $\delta_{a} g=|\lambda|^{p} g$ and so

$$
a^{-N} \delta_{a^{-1}} \widehat{g}=\widehat{\delta_{a} g}=|\lambda|^{p} \widehat{g}
$$

which entails that $a^{N}|\lambda|^{p}$ is an eigenvalue of the dilation $\delta_{a^{-1}}$ on $C_{0}\left(\mathbb{R}^{N}\right)$, a contradiction.

Theorem 3. Let $\delta_{a}$ be any nontrivial dilation. Then the following assertions hold.

i. If $\|\cdot\|$ is a complete norm on $C_{0}\left(\mathbb{R}^{N}\right)$ with the property that $\left\|\delta_{a} f\right\|=\|f\|$ for each $f \in C_{0}\left(\mathbb{R}^{N}\right)$, then $\|\cdot\|$ is equivalent to the norm $\|\cdot\|_{\infty}$.

ii. If $\|\cdot\|$ is a complete norm on $L^{p}\left(\mathbb{R}^{N}\right)$ with $1<p<\infty$ with the property that $\left\|\delta_{a} f\right\|=a^{-\frac{N}{p}}\|f\|$ for each $f \in L^{p}\left(\mathbb{R}^{N}\right)$, then $\|\cdot\|$ is equivalent to the norm $\|\cdot\|_{p}$.

Proof. In order to prove the first assertion we apply Theorem 2 to the identity operator $\Phi$ from $\left(C_{0}\left(\mathbb{R}^{N}\right),\|\cdot\|\right)$ onto $\left(C_{0}\left(\mathbb{R}^{N}\right),\|\cdot\|_{\infty}\right)$. It follows that $\mathfrak{S}(\Phi)$ is the direct sum of a finite number of scalar $\mathbb{Z}$-submodules of $C_{0}\left(\mathbb{R}^{N}\right)$. According to Lemma 2, we have $\mathfrak{S}(\Phi)=0$, and consequently $\Phi$ is continuous. This implies that $\|\cdot\|$ is equivalent to $\|\cdot\|_{\infty}$.

In the same manner we can prove the second assertion.

\section{Nonuniqueness OF NORM ON $L^{\infty}\left(\mathbb{R}^{N}\right)$}

We begin by observing that

$$
\left\|\delta_{a} f\right\|_{\infty}=\|f\|_{\infty}
$$

for all $a>0$ and $f \in L^{\infty}\left(\mathbb{R}^{N}\right)$. In this section we prove that there are other norms on $L^{\infty}\left(\mathbb{R}^{N}\right)$ enjoying that property, and therefore dilations do not determine the norm $\|\cdot\|_{\infty}$ even in the weaker sense of Theorem 3. A crucial fact in our procedure is the existence of a discontinuous linear functional $\phi$ on $L^{\infty}\left(\mathbb{R}^{N}\right)$ that is translation invariant in the sense that $\phi\left(\tau_{u} f\right)=\phi(f)$ for all $f \in L^{\infty}\left(\mathbb{R}^{N}\right)$ and $u \in \mathbb{R}^{N}$. Here 
$\tau_{u} f$ stands for the translate of $f$ defined by $\left(\tau_{u} f\right)(x)=f(x-u)$ for each $x \in \mathbb{R}^{N}$. The existence of such a functional is derived from the following.

Theorem 4 ([9, Theorem 6]). Let $G$ be a noncompact locally compact abelian group. Then both $C(G)$ and $L^{\infty}(G)$ have discontinuous translation invariant linear functionals.

Theorem 5. There exists a complete norm $\|\cdot\|$ on $L^{\infty}\left(\mathbb{R}^{N}\right)$ with the property that $\left\|\delta_{a} f\right\|=\|f\|$ for all $a>0$ and $f \in L^{\infty}\left(\mathbb{R}^{N}\right)$ and that it is not equivalent to the norm $\|\cdot\|_{\infty}$.

Proof. We begin by proving that there exists a discontinuous linear functional $\psi$ on $\left(L^{\infty}\left(\mathbb{R}^{N}\right),\|\cdot\|_{\infty}\right)$ that is dilation invariant in the sense that $\psi\left(\delta_{a} f\right)=\psi(f)$ for all $f \in L^{\infty}\left(\mathbb{R}^{N}\right)$ and $a>0$. On account of Theorem 4 , there exists a discontinuous translation invariant linear functional $\phi$ on $\left(L^{\infty}\left(\mathbb{R}^{N}\right),\|\cdot\|_{\infty}\right)$. We now consider the continuous linear operator $\Psi$ from $\left(L^{\infty}\left(\mathbb{R}^{N}\right),\|\cdot\|_{\infty}\right)$ into itself given by

$$
\Psi(f)\left(x_{1}, \ldots, x_{N}\right)=f\left(e^{x_{1}}, \ldots, e^{x_{N}}\right)
$$

for all $f \in L^{\infty}\left(\mathbb{R}^{N}\right)$ and $x_{1}, \ldots, x_{N} \in \mathbb{R}^{N}$. Set $\psi=\phi \circ \Psi$. It is easily seen that $\psi\left(\delta_{a} f\right)=\psi(f)$ for all $f \in L^{\infty}\left(\mathbb{R}^{N}\right)$ and $a>0$. On the other hand, if $f \in L^{\infty}\left(\mathbb{R}^{N}\right)$, then the function $g: \mathbb{R}^{N} \rightarrow \mathbb{C}$ defined by $g\left(x_{1}, \ldots, x_{N}\right)=f\left(\ln \left(x_{1}\right), \ldots, \ln \left(x_{N}\right)\right)$ if $x_{1}, \ldots, x_{N}>0$ and $g\left(x_{1}, \ldots, x_{N}\right)=0$ elsewhere lies in $L^{\infty}\left(\mathbb{R}^{N}\right)$ and $\Psi(g)=f$. Hence $\Psi$ is onto and so it is open. The openness of $\Psi$ together with the discontinuity of $\phi$ entails that $\psi$ is discontinuous, as required.

We now proceed to define a complete norm $\|\cdot\|$ on $L^{\infty}\left(\mathbb{R}^{N}\right)$ with the property that $\left\|\delta_{a} f\right\|=\|f\|$ for all $a>0$ and $f \in L^{\infty}\left(\mathbb{R}^{N}\right)$ and that it is not equivalent to the norm $\|\cdot\|_{\infty}$. To this end we take $\alpha \in \mathbb{C}$ with $\alpha \neq 0, \psi(\mathbf{1})$, and we note that the map $f \mapsto \alpha f-\psi(f) \mathbf{1}$ is a discontinuous linear bijection from $\left(L^{\infty}\left(\mathbb{R}^{N}\right),\|\cdot\|_{\infty}\right)$ onto itself. Consequently, the map

$$
\|f\|=\|\alpha f-\psi(f) \mathbf{1}\|_{\infty}
$$

gives a complete norm on $L^{\infty}\left(\mathbb{R}^{N}\right)$ that is not equivalent to $\|\cdot\|_{\infty}$. For all $f \in$ $L^{\infty}\left(\mathbb{R}^{N}\right)$ and $a>0$, we have

$$
\begin{aligned}
\left\|\delta_{a} f\right\| & =\left\|\alpha \delta_{a} f-\psi\left(\delta_{a} f\right) \mathbf{1}\right\|_{\infty}=\left\|\alpha \delta_{a} f-\psi(f) \mathbf{1}\right\|_{\infty} \\
& =\left\|\delta_{a}(\alpha f-\psi(f) \mathbf{1})\right\|_{\infty}=\|\alpha f-\psi(f) \mathbf{1}\|_{\infty} \\
& =\|f\|,
\end{aligned}
$$

as required.

\section{REFERENCES}

[1] H. G. Dales, Banach algebras and automatic continuity. London Mathematical Society Monographs, New Series, 24, The Clarendon Press, Oxford University Press, New York, 2000. MR 2002e: 46001

[2] J. Extremera, J. F. Mena, and A. R. Villena, Uniqueness of the topology on $L^{1}(G)$, Studia Math. 150 (2002), 163-173. MR 2004a:43003

[3] J. Extremera, J. F. Mena, and A. R. Villena, Uniqueness of norm on $L^{p}(G)$ and $C(G)$ when $G$ is a compact group. J. Funct. Anal. 197 (2003), 212-227.

[4] J. Extremera and A. R. Villena, Uniqueness of norm on $L^{1}(G)$ when $G$ is a Moore group, preprint, 2002.

[5] K. Jarosz, Uniqueness of translation invariant norms, J. Funct. Anal. 174 (2000), 417-429. MR 2001e: 46092 
[6] B. E. Johnson, The uniqueness of the (complete) norm topology, Bull. Amer. Math. Soc. 73 (1967), 537-539. MR 35:2142

[7] B. E. Johnson, Continuity of homomorphisms of Banach G-modules, Pacific J. Math. 120 (1985), 111-121. MR 87c:46057

[8] A. R. Villena, Uniqueness of the topology on spaces of vector-valued functions, J. London Math. Soc. 64 (2001), 445-456. MR 2002g:46056

[9] G. S. Woodward, Translation-invariant linear forms on $C_{0}(G), C(G), L^{p}(G)$ for noncompact groups, J. Funct. Anal. 16 (1974), 205-220. MR 49:9540

Departamento de Análisis Matemático, Facultad de Ciencias, Universidad de Granada, 18071 Granada, Spain

Departamento de Análisis Matemático, Facultad de Ciencias, Universidad de Granada, 18071 Granada, Spain

E-mail address: avillena@ugr.es 